\title{
Application of Machine Learning in Cricket and Predictive Analytics of IPL 2020
}

\author{
Anurag Sinha \\ Jharkhand \\ Department of computer science and IT, Student, Amity University Jharkhand Ranchi,
} (India), 834001

anuragsinha257@gmail.com

\begin{abstract}
In cricket, particularly the twenty 20 format is most watched and loved by the people, where no one can guess who will win the match until the last ball of the last over. In India, The Indian Premier League (IPL) started in 2008 and now it is the most popular T20 league in the world. So we decided to develop a machine learning model for predicting the outcome of its matches. Winning in a Cricket Match depends on many key factors like a home ground advantage, past performances on that ground, records at the same venue, the overall experience of the players, record with a particular opposition, and the overall current form of the team and also the individual player. This paper briefs about the key factors that affect the result of the cricket match and the regression model that best fits this data and gives the best predictions. Cricket, the mainstream and widely played sport across India which has the most noteworthy fan base. Indian Premier League follows 20-20 format which is very unpredictable. IPL match predictor is a ML based prediction approach where the data sets and previous stats are trained in all dimensions covering all important factors such as: Toss, Home Ground, Captains, Favorite Players, Opposition Battle, Previous Stats etc, with each factor having different strength with the help of KNIME Tool and with the added intelligence of Naive Bayes network and Eulers strength calculation formula.
\end{abstract}

Keywords: Naïve Bayes Classification, Eulers Strength Formula, Cricket Prediction, Supervised Learning, KNIME Tool, Cricket prediction, sports analytics, multivariate regression, neural network

\section{Applications}

The main objective of sports prediction is to improve team performance and enhance the chances of winning the game. The value of a win takes on different forms like trickles down to the fans filling the stadium seats, television contracts, fan store merchandise, parking, concessions, sponsorships, enrollment and retention.

\section{Data}

Real world data is dirty. We can't expect a nicely formatted and clean data as provided by Kaggle. Therefore, data pre-processing is so crucial that I can't stress enough how important it is. It is the most important stage as it could occupy $40 \%-70 \%$ of the whole workflow, just to clean the data to be fed to your models.

I scraped three scripts from Crickbuzz website comprising of rankings of teams as of May 2019, details of the fixtures of 2019 world cup and details of each team's history in previous world cups. I stored the above piece of data in three separate csv files. For the fourth file, I grabbed odi data-set for matches played between 1975 and 2017 from Kaggle in another csv file. In this file, I removed all the data from 1975 to 
2010. This was done as the results of the last few years should only matter for our predictions. Since I didn't get the data for 2018 and 2019 so this model might not be that accurate but still I believe this gives a fairly good idea. Then I did manual cleaning of the data as per my needs to make a machine learning model out of it.

\section{INTRODUCTION}

After football, cricket is most loved and watched by many individuals in the world but in India cricket is the most loved sport. In the past few years, lots of research papers are published and lots of work is done which predicts the result of a cricket match by using the factors that affect the match outcome and they are using the supervised machine learning algorithms to predict the outcome of the match like Linear regression, support vector machines, logistic regression, decision tree, Bayes network, random forest. Cricket is one of the most well-liked sports in the world. Especially the Twenty 20 format is very popular as it is a fast-paced form of the game that attracts the spectators at the ground and the viewers at home. The Indian Premier League (IPL) is a professional Twenty 20 cricket league that is governed by the Board of Control for Cricket in India (BCCI). The Indian Premier League is conducted every year and participating teams represent a city in India. Various natural factors affect the game, the hype has given by the media, and a huge market like fantasy 11 and betting on sites has provided a lot of importance to the model. The rules of the game, the skill of the players, their form, and various other natural factors are very important in the prediction accuracy of the result of a cricket match. As the technology is growing and the apps like fantasy 11 and betting sites are getting popular the people going to use the predictions given by the machine learning model. The use of machine learning makes life easier in many aspects. To predict the outcome of a cricket match we are not going to rely on a single machine learning algorithm we are going to use all the machine learning algorithms. In machine learning, there are two types of learning supervised learning and unsupervised learning. In Unsupervised learning, the data is not properly labelled so the machine has to sort the data according to patterns, combinations without any training given. But in supervised learning, the data is labelled with the proper classification so the machine can easily analyze it and produce the correct result. For our application, the unsupervised learning models are not of any use because the data of cricket matches are properly labelled. So we are going to use the supervised learning models. In Supervised learning, there are again two types are classification and regression. Classification is used to classify among categories like red or blue and Regression is used when the output is a real number like rupees or height. In our model, we are going to use regression because the outcome will be the winning percentage and it is of type number. Our main objective is to find the key factors that affect the match outcome and select the best machine learning model that best fits this data and gives the best results. Some works already have been published in this area of predicting the outcome of a cricket match. In some paper, only a few key factors are taken for prediction so the accuracy is less. Whereas in some paper the machine learning model is not appropriate. So it is important to take all the key factors that can affect the match outcome and as well as to select the best model for training and testing the data. This will increase the prediction accuracy drastically.

\section{LITERATURE REVIEW}

An extensive online search produced very few articles related to players' performance prediction in the game of cricket. A very small number of researchers have studied the performance of cricket players. Muthuswamy and Lam [1] predicted the performance of Indian bowlers against seven international teams against which the Indian cricket team plays most frequently. They used back propagation network and radial basis network function to predict how many runs a bowler is likely to concede and how many 
wickets a bowler is likely to take in a given ODI match. Wikramasinghe [2] predicted the performance of batsmen in a test series using a hierarchical linear model. Barr and Kantor [3] defined a criterion for comparing and selecting batsmen in limited overs cricket. They defined a new measure $\mathrm{P}$ (out) i.e. probability of getting out and used a two-dimensional graphical representation with Strike Rate on one axis and $\mathrm{P}$ (out) on another. Then they define a selection criterion based on $\mathrm{P}($ out $)$, strike rate and batting average of the batsmen. Iyer and Sharda [4] used neural networks to predict the performance of players where they classify batsmen and bowlers separately in three categories - performer, moderate and failure. Based on the number of times a player has received different ratings, they recommend if the player should be included in the team to play World Cup 2007. Jhanwar and Paudi [5] predict the outcome of a cricket match by comparing the strengths of the two teams. For this, they measured the performances of individual players of each team. They developed algorithms to model the performances of batsmen and bowlers where they determine the potential of a player by examining his career performance and then his recent performances. Lemmer [6] defined a new measure called Combined Bowling Rate to measure the performance of bowlers. The combined bowling rate is a combination of three traditional bowling measures: bowling average, strike rate and economy. Bhattacharjee and Pahinkar. [7] used this combined bowling rate to analyze the performance of bowlers in Indian Premier League (IPL). They also determined other factors that affect the performance of bowlers and applied multiple regression model to identify the factors that are empirically responsible for the performance of bowlers. Mukharjee. [8] applied Social Network Analysis to rate batsmen and bowlers in a team performance. He generated a directed and weighted network of batsmen-bowlers using player-vs-player information available for test and ODI cricket. He also generated a network of batsmen and bowlers using the dismissal record of batsmen in the history of cricket. Shah [9] also defined new measures to measure players' performance. The new measure for batsmen takes into account the quality of each bowler he is facing and the new measure for bowlers considers the quality of each batsman he is bowling to. The aggregate of individual performance of a batsman against each bowler is the total performance index of the batsman. Similarly, the aggregate of individual performance of a bowler against each batsman is the total performance index of the bowler. Parker, Burns and Natarajan. [10] defined a model for valuation of players for IPL auction. Their model considered factors like previous bidding price of the player, experience of the player, strike rate etc. Prakash, Patvardhan. and Lakshmi [11] defined batting index and bowling index to rank players' performance for their models to predict outcomes of IPL matches. Ovens and Bukiet [12] applied a mathematical approach to suggest optimal batting orders for ODI matches. Our work is probably the first generalized approach to predict how many runs will a batsman score and how many wickets will a player take on a particular match day. Muthuswamyand Lam [1] carried out a similar study predicting how many wickets will a bowler take using neural networks but their work was limited to eight Indian bowlers and is difficult to generalize for all the bowlers in the world. We used some supervised machine learning algorithms to build prediction models that can be used to predict the performance of any player in a given match.

A. Rabindra Lamsal and Ayesha Choudhary [1] In this paper, they have taken the data of matches from the official website of the Indian Premier League. That data had many features so they analyzed the data and selected some key features. They had used the scikit-learn machine learning library to pre-process the data and applied some selection models. They removed the low variance, univariate and recursive features. Byusing these feature selection models they found 5 key features from 15 . The features are home team, away team, venue, toss winner, toss decision and winner. They had trained the Random Forests and Multiple Linear Regression model by 10 seasons of IPL data and trained by the 11 th season's data. 41 out of 60 matches their model able to classify correctly. So their accuracy is $68.33 \%$ which is not so good. Limitations of this model are it uses only 5 features and only 2 machine learning models. B. Abhishek Naik, Shivanee Pawar, Minakshee Naik, Sahil Mulani [2] This paper processes the data dynamically and gives a prediction as the match progresses. Before the match starts their prediction depends on the factors like batting, bowling, batting order, captain of both the teams and batting-bowling stats on that ground 
against that opponent and after the match starts their prediction depends on batsman-bowler performance and battingbowling order of particular player. They are predicting only the one day international (ODI) matches by using the logistic regression and K-means clustering. In this paper, they had only tested this model on one match which is India vs Australia happed on 26th March 2015 at Sydney cricket ground and their prediction was correct. Their predictions can go wrong sometimes because they are fluctuating on every ball. C. Singhvi, Arjun, Ashish Shenoy, Shruthi Racha and Srinivas Tunuguntla [3] In this paper, they have taken 16 features to train the model. Features are like average runs scored by a player, the average number of $4 \mathrm{~s}$ and $6 \mathrm{~s}$ hit by a player, average strike rate of a player, number of times the player is not out, numbers of the $50 \mathrm{~s}$ and $100 \mathrm{~s}$ scored by the player, total number of matches played by a player, current and average batting position, average number of wickets taken in a match by bowler, average economy and average runs conceded, average number of wide and no-balls bowled and last is average number of maiden overs bowled. They had taken the data of all T20 matches domestic, league matches and international. Many machine learning algorithms are used like Randomizes Forest, Naive Bayes, Decision Trees, Linear SVM, Non-Linear SVM and they are trained by data of 5390 T20 matches. After testing the model the Support Vector Machine given the best accuracy of prediction which is $63.89 \%$. D. Swetha, Saravanan.KN [4] In This paper only briefs about the key factors that cricket match depends on. No machine learning model is trained to predict the match result. The factors discussed in this paper are pitch, toss, and team strength, past records, home ground advantage, current performance, and weather. Pitch plays a very important role in the match because how the ball will behave is totally dependent on it. Toss is also important the teams chasing first wins more matches as the target is known and dew comes in play after evening. By calculating the average of all players and the current form of players we can easily find out the team strength. Past performances play a vital role in prediction, what is the performance of a team on the ground against a particular opponent is very important. If a team is playing on the home ground then crowd support becomes the 12th man of the team also the players are familiar with the playing conditions. The current form of the team and players is also important to predict the winner. The weather condition also affects the swing of the ball and the match outcome. These features can be used to train the model to get better prediction accuracy. E. Geddam Jaishankar Harshit, Rajkumar S [5] This paper compares various supervised machine learning algorithms that can be used to predict the match result. A dataset of 5000 one day international matches is taken from Cricinfo and 70\% is used to train the model and $30 \%$ is used to test the model. They are using Support Vector Machine, Logistic Regression, Decision Tree and Bayes Classifier as machine learning algorithms. They got $60 \%$, $65 \%, 67 \%$ and $72 \%$ respectively. So as we can see the Bayes classifier has the best accuracy among all. Analytics in Cricket

\section{Dhoni vs Kohli |Cricket Analytics Part 3 - Drawing Useful Inferences from Machine Learning}

In the final post of the series, we will make use of Machine Learning techniques for complex analysis. Before explaining how to implement this in $\mathrm{R}$, we would like to emphasize the importance of machine learning in sports especially cricket.

Machine Learning(ML) has brought about a positive change in most of the fields. It can also be applied in sports like cricket.ML can improve the performance and accuracy of players and develop better strategies for the upcoming games. This can be done by predicting the runs scored by a player or the team, the wickets that can be taken and finally predicting the final result of the match. It is always important to select the correct variables so that the prediction is accurate. In the post we will focus on applying ML techniques to the dataset in R. As we are dealing the batting dataset of Virat Kohli and MS Dhoni, we will be dealing with the following variables:

- Runs which indicates the runs scored by the player.

- $\mathrm{BF}$ which indicates the balls faced by the player. 
- Mins which indicates the minutes spent at the crease by the player.

Before proceeding with the complex analysis it is important that we extract and clean the data.

\section{Holt-Winters Model for predicting the runs that can be scored}

The batsmanPerfForecast function is available in cricketr package.This function is used for predicting the performance of the player in terms of runs. The parameters that need to be passed are:

- file which indicates the file name.Here, kohliOd and dhoniOd are the file names of Virat and Dhoni respectively.

- name which indicates the name of the player whose performance needs to be predicted.

The working of the function is explained below :

Based on the total number of rows available in the dataset the training and the testing data are divided in 90:10 ratio. The model used here to fit the training data is Holt-Winters Model. This model is used for predicting the runs by minimizing the squared error of prediction. It works on the basic principle of time series forecasting. Time series forecasting makes use of previous month's or day's or year's data to forecast the value for future. The starting month and the corresponding year and the ending month and the corresponding year (which is at 0.9 of the length of the rows) is the range for which the runs are taken for the training dataset. The similar procedure is followed for the rest of the $10 \%$ runs for creating a testing set. Then the Holt-Winters Model is trained using this data and the data forecasted is stored separately. This model is mostly employed in those situations that show a pattern repeating over some time i.e- a trend, a season or both. The usual forecasting methods require the weighted average of the data. Whereas this model forecasts by associating the data with exponential weighted moving average. This method is also called Triple exponential smoothing because of its three parameters:

1. Alpha parameter: This estimates the level component at the current point of time.

2. Beta parameter: This estimates the trend component at the current point of time. This can be seen by the slope.

3. Gamma parameter: This estimates the seasonal component at the current point of time.

The above three parameters have a value between 0 and 1 . If the values are close to zero then it means that the recent data points have been given less weight in comparison to the old data points while making the predictions.

After all the data has been generated it is plotted as seen below. The main features of the plot are :

- The training data is plotted as a line in magenta color.

- The testing data is plotted as a line in red color.

- The forecasted data is plotted as a line in blue color. 


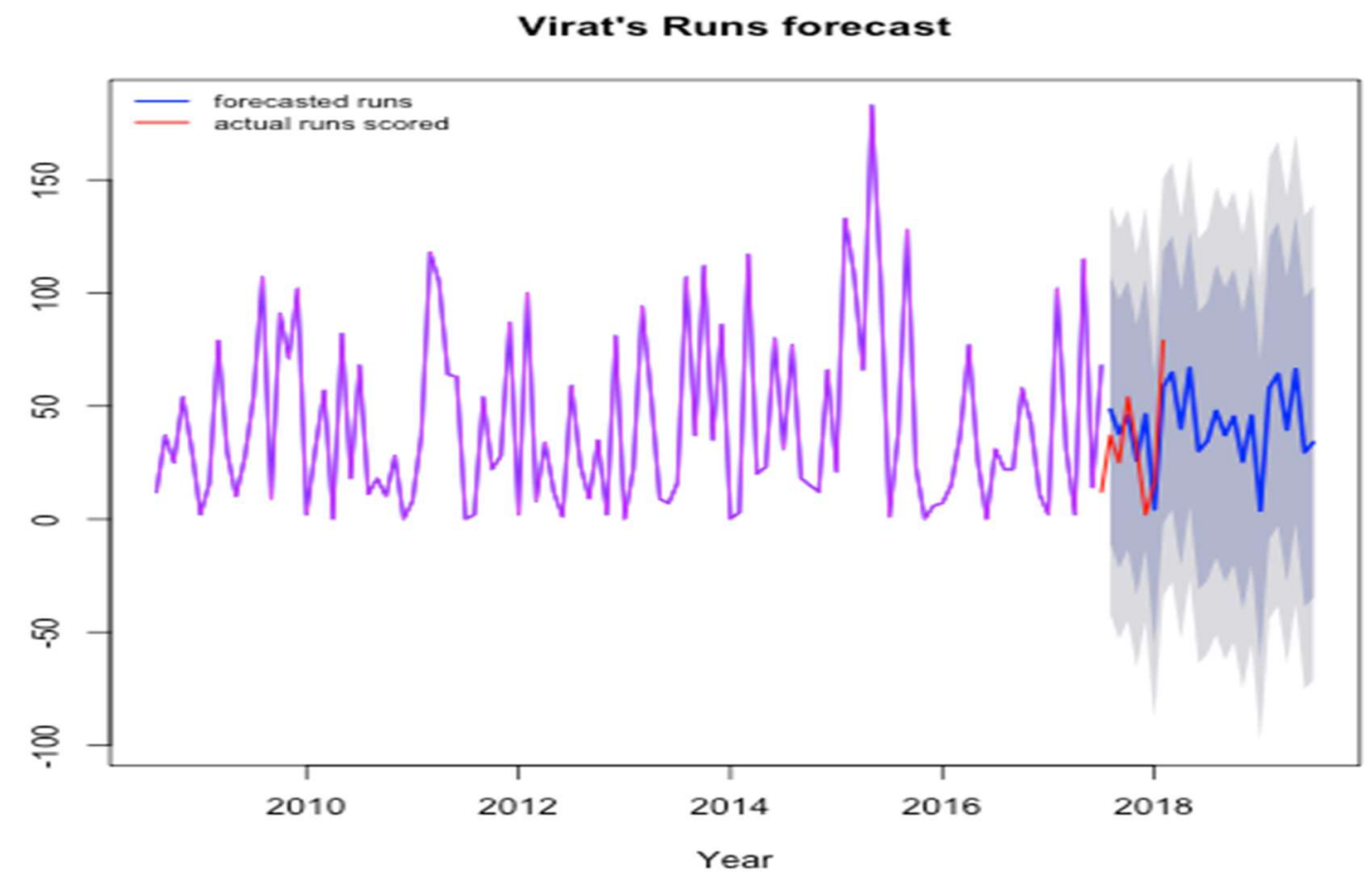

From above we can observe the following:

- The value of alpha is 0.06946173 which is very low indicating that value of the level component at the current point of time relies less on recent and more on the past data.

- The value of beta is 0.04646374 which is very low indicating that value of the trend component at the current point of time relies less on recent and more on the past data. From the plot below you can observe that slope doesn't change a lot.

- The value of gamma is 0.2026552 which is low indicating that value of the seasonal component at the current point of time relies less on recent and more on the past data.

The list of coefficients specifies the value of the level, trend and seasonal component for each month. 


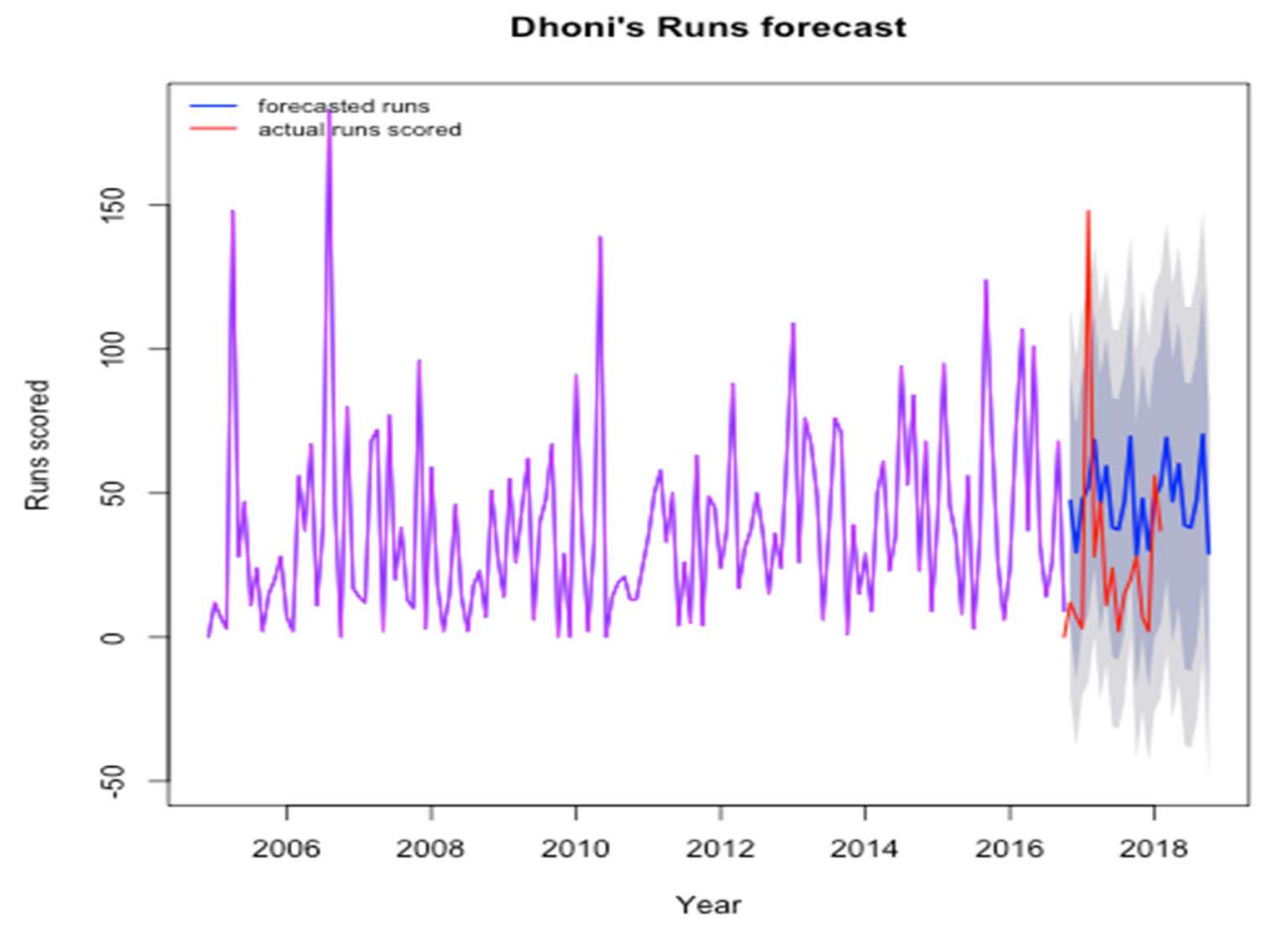

K Means for predicting player's runs scoring likelihood in \%

Next we have used the batsmanRunsLikelihood function available in cricketr package.This function is used for predicting the runs likelihood in terms of percentage, based on the balls faced and the minutes spent at the crease of Kohli. The parameters that need to be passed are:

- file which indicates the file name.Here, kohliOd and dhoniOd are the file names of Virat and Dhoni respectively.

- name which indicates the name of the player whose performance needs to be predicted.

The working of the function is explained below :

The function makes use of $\mathrm{K}$ Means Clustering technique. It is an unsupervised technique used for grouping similar instances together in a cluster. The number of the clusters to be formed needs to specified. Here the number of clusters formed is three. The kmeans function is used for getting the three centroids of the three clusters. The below plot is a 3D scatterplot having the centroid of the clusters plotted. The three centroids are colored in red, blue and black. The three axes are as follows:

- The $\mathrm{x}$-axis indicates the number of balls faced.

- The y-axis indicates the minutes spent at the crease.

- The z-axis indicates the runs scored by the player.

The significance of three clusters is to emphasize on the three phases of the batsman that is the beginning, the middle and the end of the innings. The first cluster formed is closer to the origin, the second one somewhere in the middle and the third cluster is far away from the origin. The percentage likelihood in the summary is calculated by dividing the sum of the data points in a particular cluster divided by the number of data points in all the three clusters. 


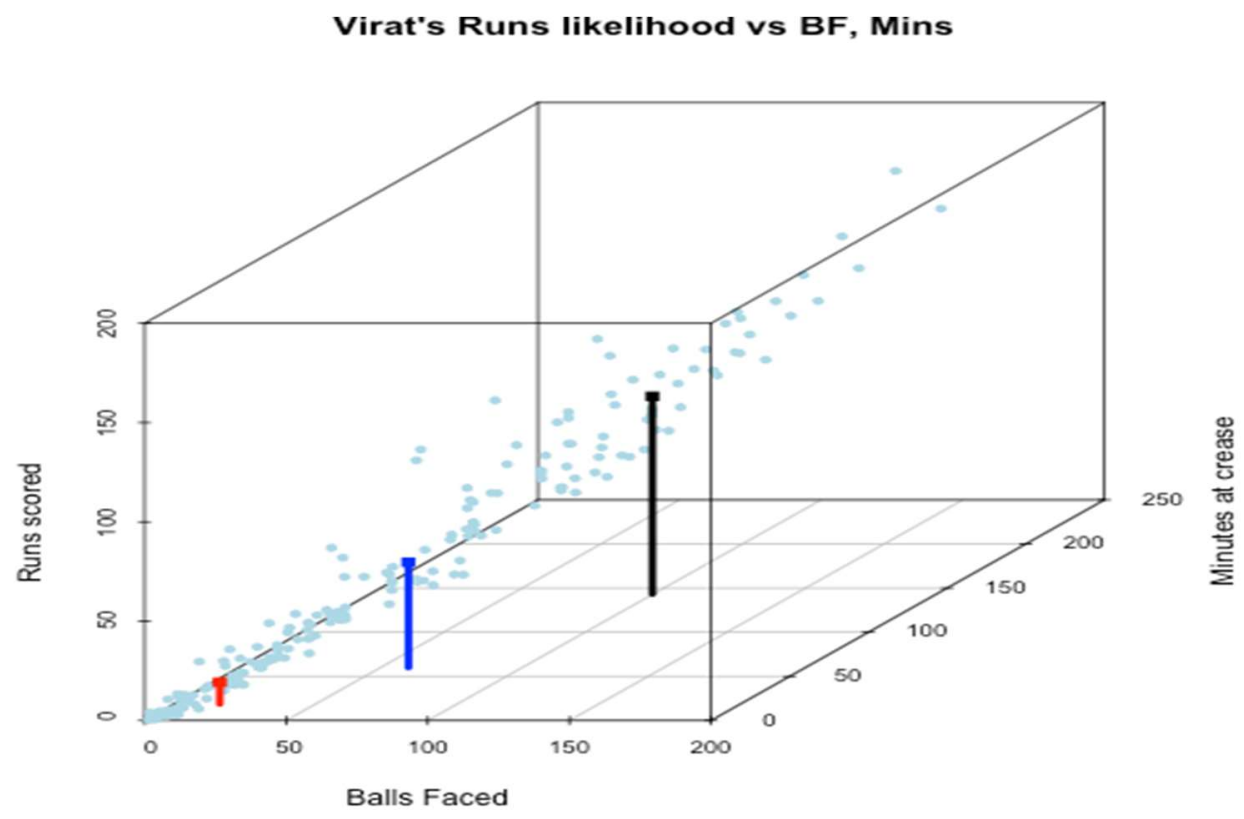

The above table gives the $\mathrm{z}, \mathrm{x}$ and $\mathrm{y}$ values of the centers (column-wise) of the three clusters(row-wise) for Dhoni.

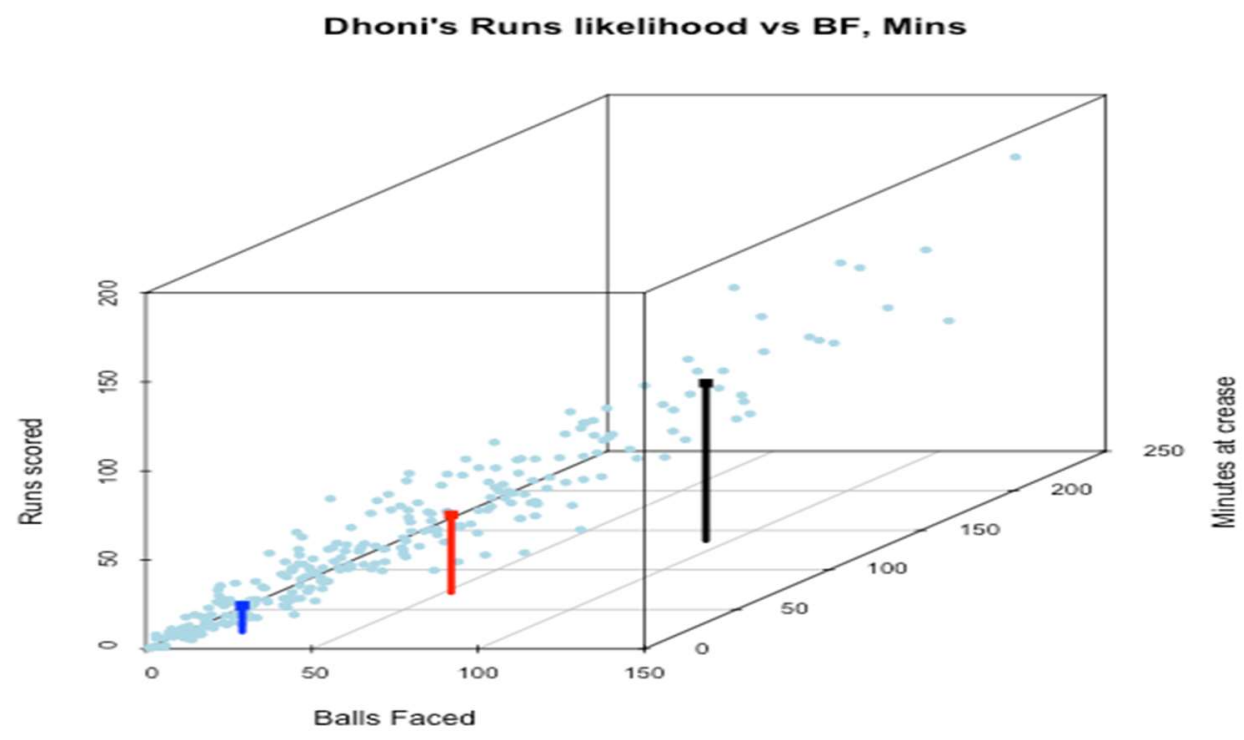

4. Process Flow The process stream shows how the entire procedure of the project is done and the result is seen. The following flow chart shows the procedure: 


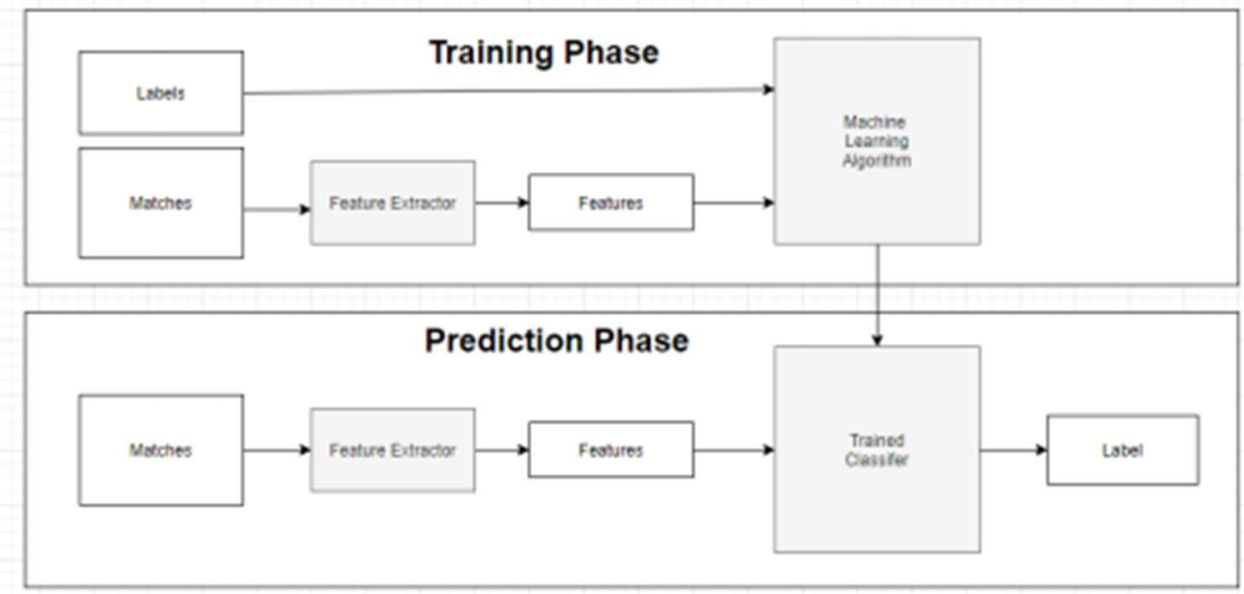

Figure 1. Process Flow

I followed the general machine learning workflow step-by-step:

1. Data cleaning and formatting.

2. Exploratory data analysis.

3. Feature engineering and selection.

4. Compare several machine learning models on a performance metric.

5. Perform hyper-parameter tuning on the best model.

6. Evaluate the best model on the testing set.

7. Interpret the model results.

8. Draw conclusions and document work.

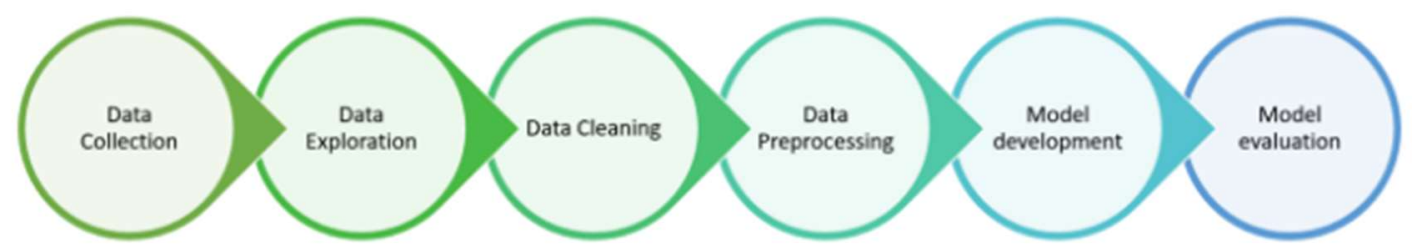

Machine learning workflow

1. Data cleaning and formatting

Next, let's display the details of matches played by India. 


\begin{tabular}{l|l|l|l|l|l|l|l|}
\hline Out [189]: & date & Team_1 & Team_2 & Winner & Margin & Ground \\
\cline { 2 - 7 } & $\mathbf{1}$ & 5-Jan-10 & India & Sri Lanka & Sri Lanka & 5 wickets & Dhaka \\
\hline $\mathbf{2}$ & 7-Jan-10 & Bangladesh & India & India & 6 wickets & Dhaka \\
\hline $\mathbf{4}$ & 10-Jan-10 & India & Sri Lanka & India & 8 wickets & Dhaka \\
\hline $\mathbf{5}$ & 11-Jan-10 & Bangladesh & India & India & 6 wickets & Dhaka \\
\hline $\mathbf{6}$ & 13-Jan-10 & India & Sri Lanka & Sri Lanka & 4 wickets & Dhaka \\
\hline
\end{tabular}

\section{Exploratory data analysis}

After that, I merged the details of the teams participating this year with their past results.

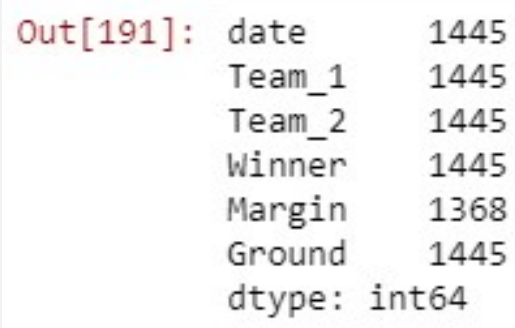

I deleted the columns like date of the match, margin of victory, and the ground on which the match was played. These features doesn't look important for our prediction.

\begin{tabular}{l|l|l|l|l|}
\hline Out[193 ]: & Team_1 & Team_2 & Winner \\
\hline 0 & Bangladesh & Sri Lanka & Sri Lanka \\
\hline $\mathbf{1}$ & India & Sri Lanka & Sri Lanka \\
\hline $\mathbf{2}$ & Bangladesh & India & India \\
\hline $\mathbf{3}$ & Bangladesh & Sri Lanka & Sri Lanka \\
\hline $\mathbf{4}$ & India & Sri Lanka & India \\
\hline
\end{tabular}

\section{Feature engineering and selection}

This is probably the most important part in the machine learning workflow. Since the algorithm is totally dependent on how we feed data into it, feature engineering should be given topmost priority for every machine learning project. 


\section{Perform hyperparameter tuning on the best model}

The popularity of the Random Forest model is explained by its various advantages:

- Accurate and efficient when running on large databases

- Multiple trees reduce the variance and bias of a smaller set or single tree

- Resistant to overfitting

- Can handle thousands of input variables without variable deletion

- Can estimate what variables are important in classification

- Provides effective methods for estimating missing data

- Maintains accuracy when a large proportion of the data is missing

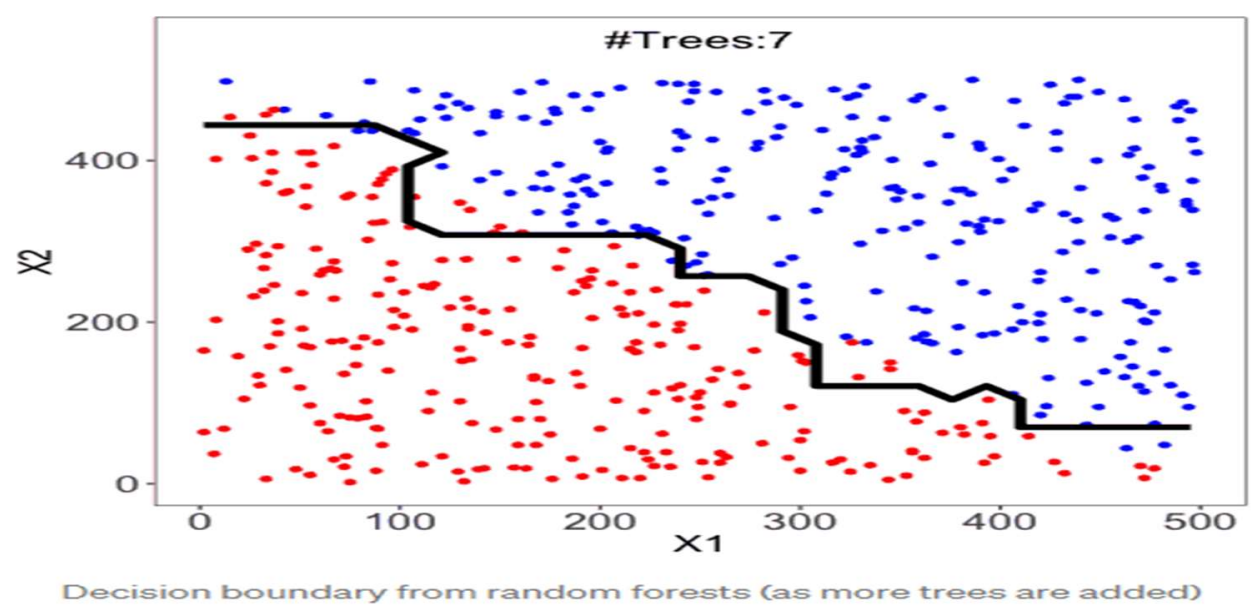

\section{Evaluate the best model on the testing set}

Let's continue. I added IPL rankings of teams giving priority to higher ranked team to win this year.

Next, I added new columns with ranking position for each team and slicing the dataset for first 45 games since there are 45 league stage games in total.

\section{Predicting IPL-2020 Winner}

Classification and Regression are the two branches of Supervised Learning in the field of Machine Learning. These are the basic topics that one should learn when starting their journey with Machine Learning. Doing projects is the only way through which one can learn and master these topics.

In my previous blog, I did a classification project. Having learnt some of the concepts in Classification, the time has arrived for me to make my hands dirty with Regression. 


\section{Dataset:}

We have scraped the data from ICC's T20 top 100 players and Cricbuzz websites using Beautiful Soup module and obtained the data for Batsmen, Bowler and All-Rounder separately.

Features used for Batsmen:

- Innings

- $\quad$ Runs Scored

- $\quad$ Batting Average

- $\quad$ Batting Strike Rate

- $\quad$ Fifties

- Fours

- Sixes

Features used for Bowlers:

- Innings

- Wickets

- Economy

- Bowling Average

- Bowling Strike Rate

Features used for All-Rounders:

- Innings as Batsmen

- Runs Scored

- Batting Average

- Batting Strike Rate

- Fifties

- Fours

- Sixes

- Innings as Bowler

- Wickets

- Economy

- Bowling Average 
- Bowling Strike Rate

Now that we have our data, it is time for us to observe and understand the data.

\section{Data Pre-Processing:}

We have plotted the histogram of the data and observed that the data is highly right skewed. To overcome the skewness, we have log-transformed the data, so that the data is now approximately normal and our models work well.

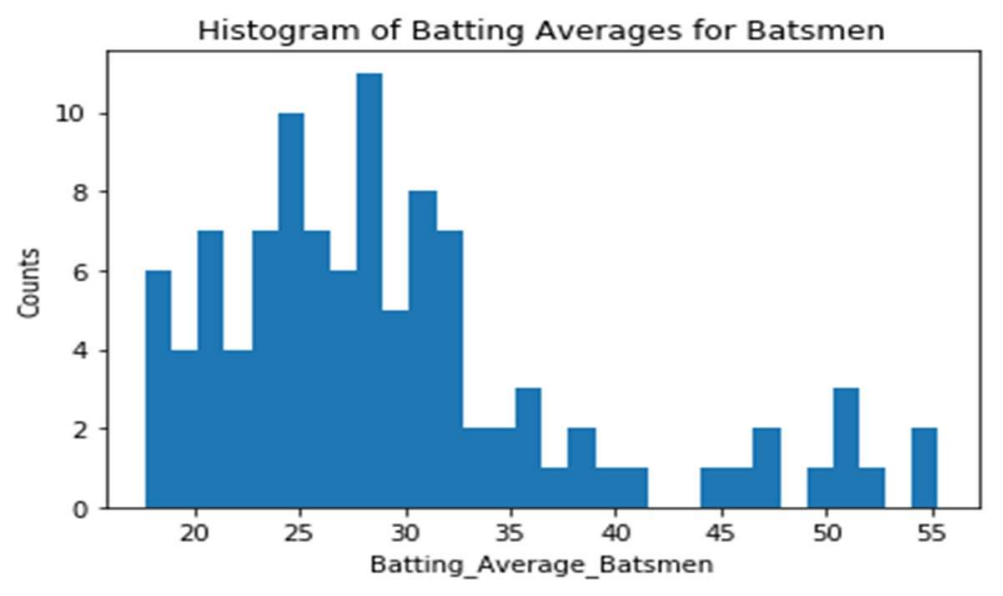

Right Skewed Data

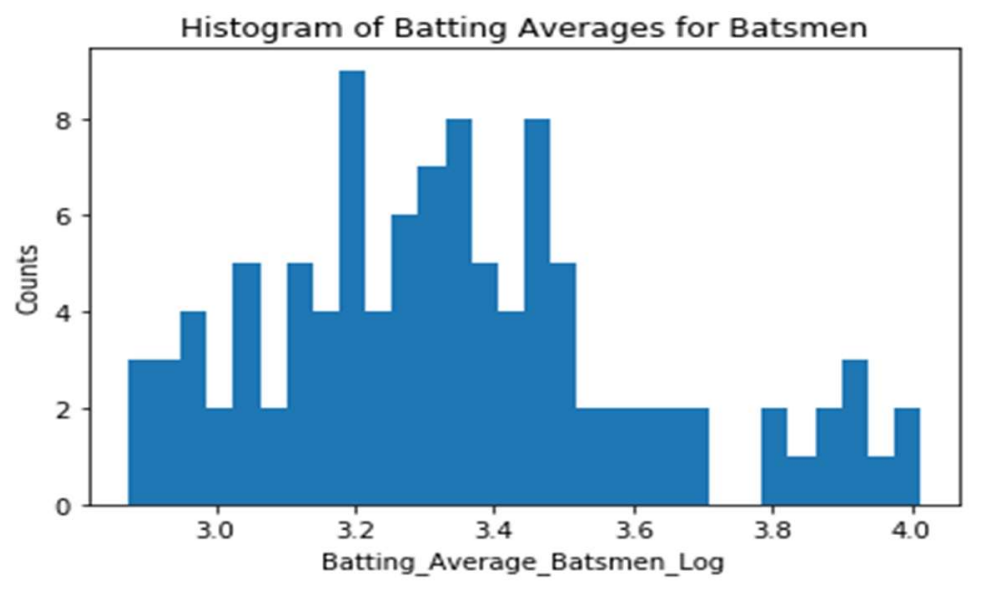

We have observed that some records have 0 values for some of the features. We have dropped those records from our datasets. Most probably they are the players who played very less number of matches.

\section{Model Building:}

Now that we have processed and cleaned our data, we have to build the machine learning models on this cleaned data. We have implemented SGDRegressor, KNN-Regressor,Linear Regression using LeastSquare Estimates, Weighted KNN-Regressor and compared our models with the Scikit learn's model and we have achieved almost similar results to that of the Scikit Implementation. 


\section{SGD-Regressor:}

We have provided the training data as the input to the models and found the best number of epoch using Cross Validation Data. AS we can see in the plots, the training error decreases as the epochs increases which means we are going towards convergence. We can increase the number of epochs to reduce the error furthermore, but we have stopped at 2000 epochs as the error fall rate is almost steady at this point.

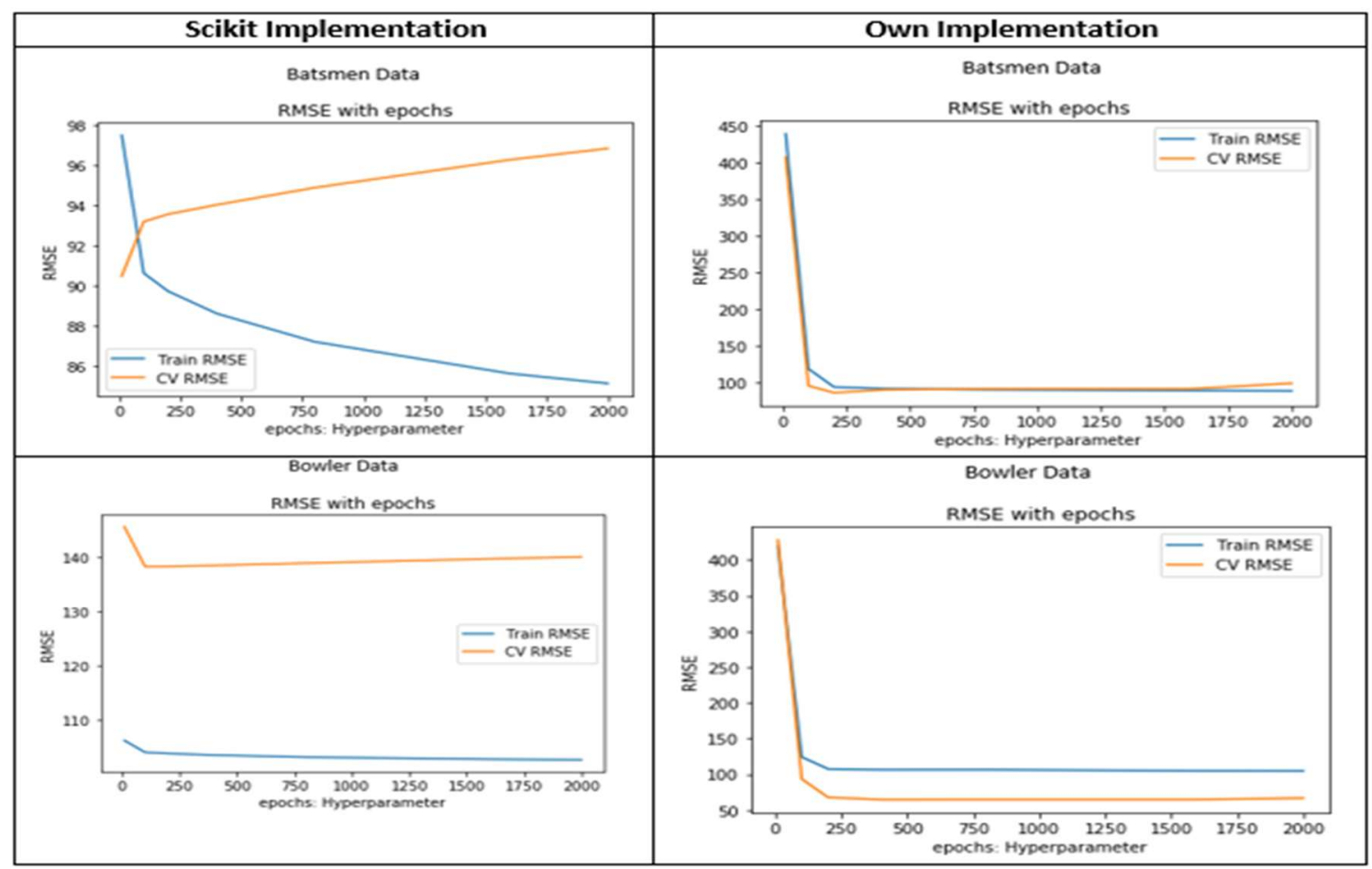

We have got the best number of epochs using cross validation. Now, we can run the model with this best number of epochs on test data and we can obtain the predicted values. We can then compare our predicted values with the actual values to get the error.

After running our model on the test data, we get the below results. 


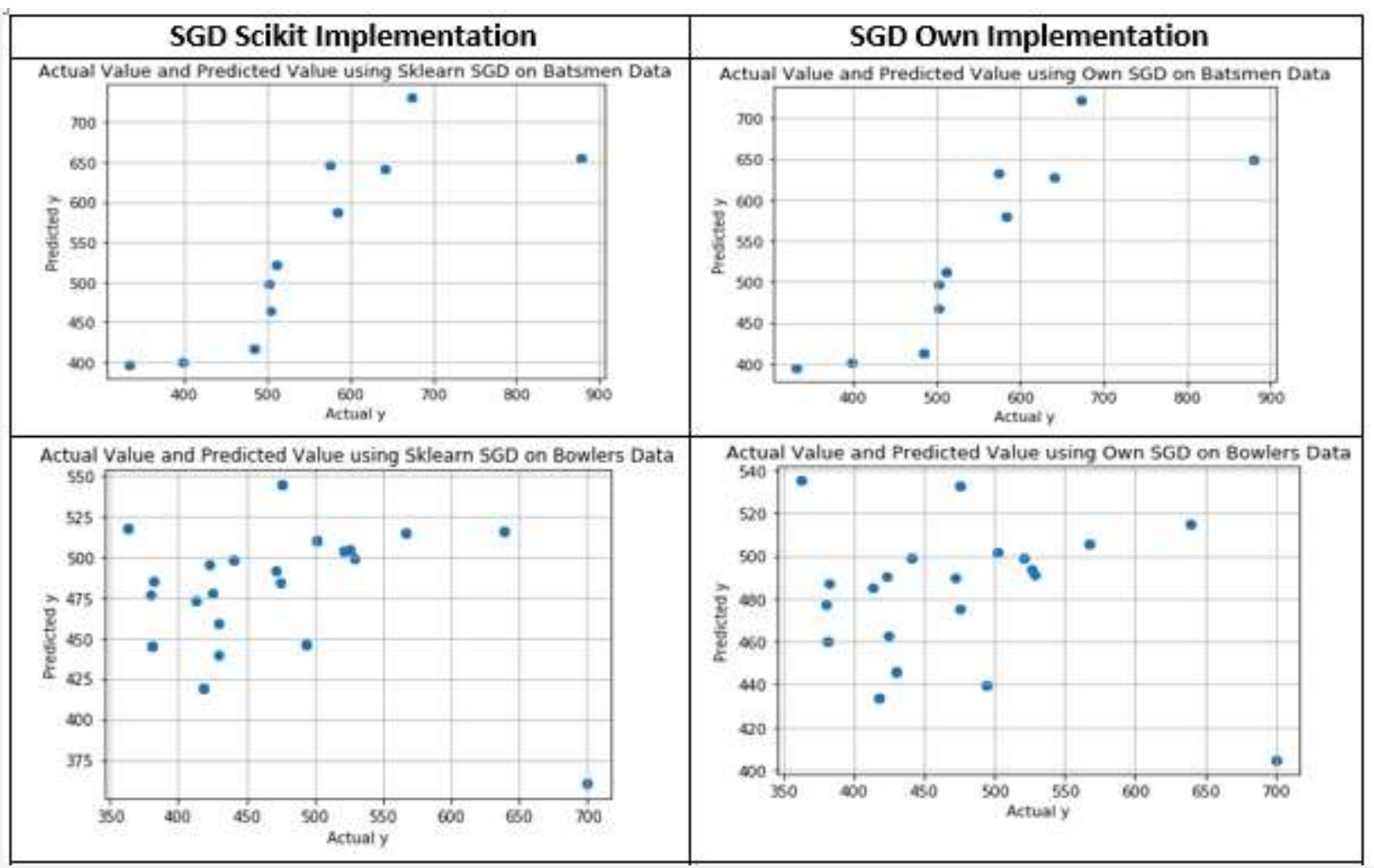

We can see that Scikit implementation is almost similar to our results.

\section{KNN-Regressor:}

We have provided the training data as the input to the models and found the best number of neighbours using Cross Validation Data. As we can see from the plots, the training error increases as the value of $\mathrm{K}$ increases, which means that smaller values of $\mathrm{K}$ leads to overfitting and larger values of $\mathrm{K}$ leads to underfitting. 


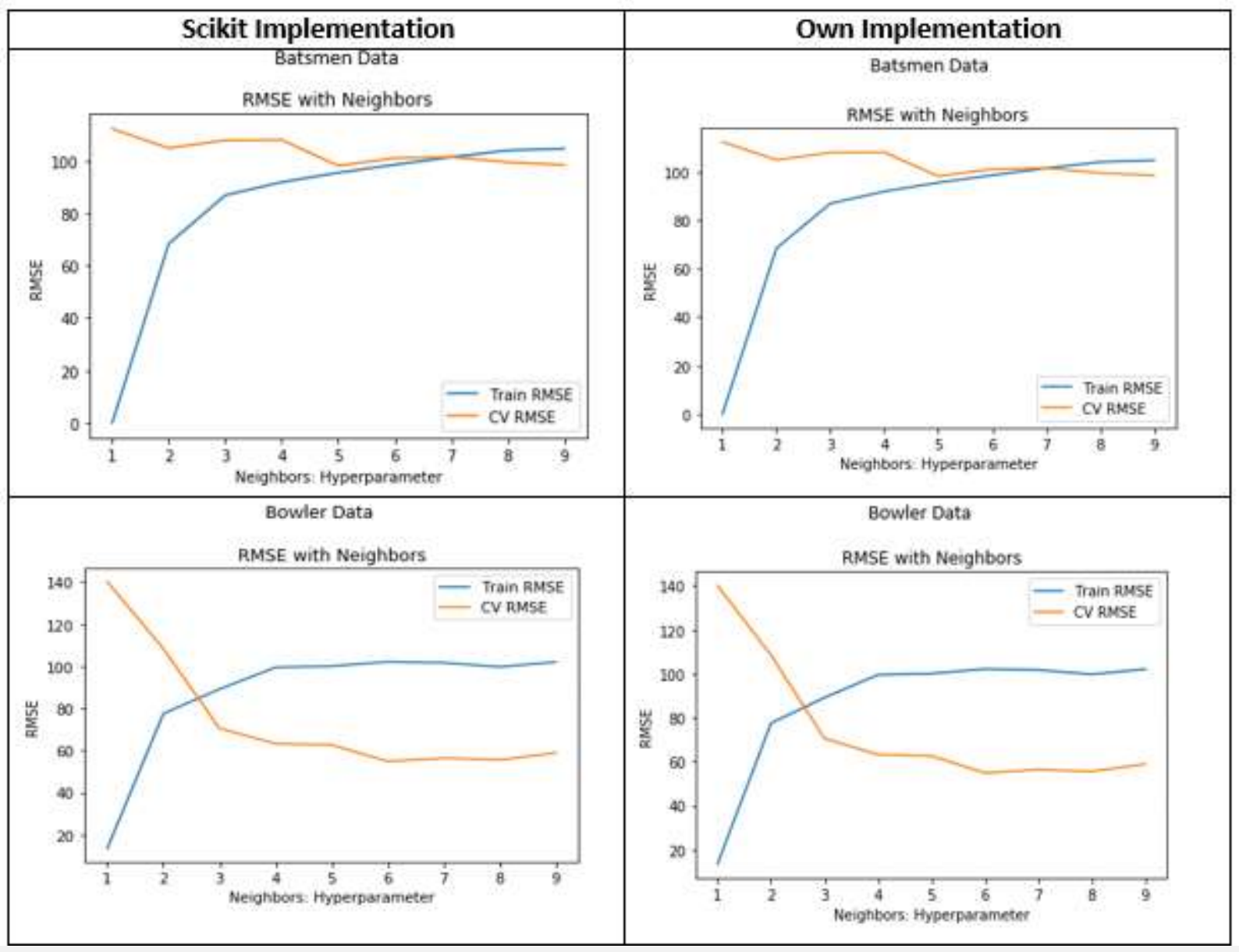

We have got the best number of neighbors using cross validation. Now, we can run the model with this best number of epochs on test data and we can obtain the predicted values. We can then compare our predicted values with the actual values to get the error.

After running our model on the test data, we get the below results. 


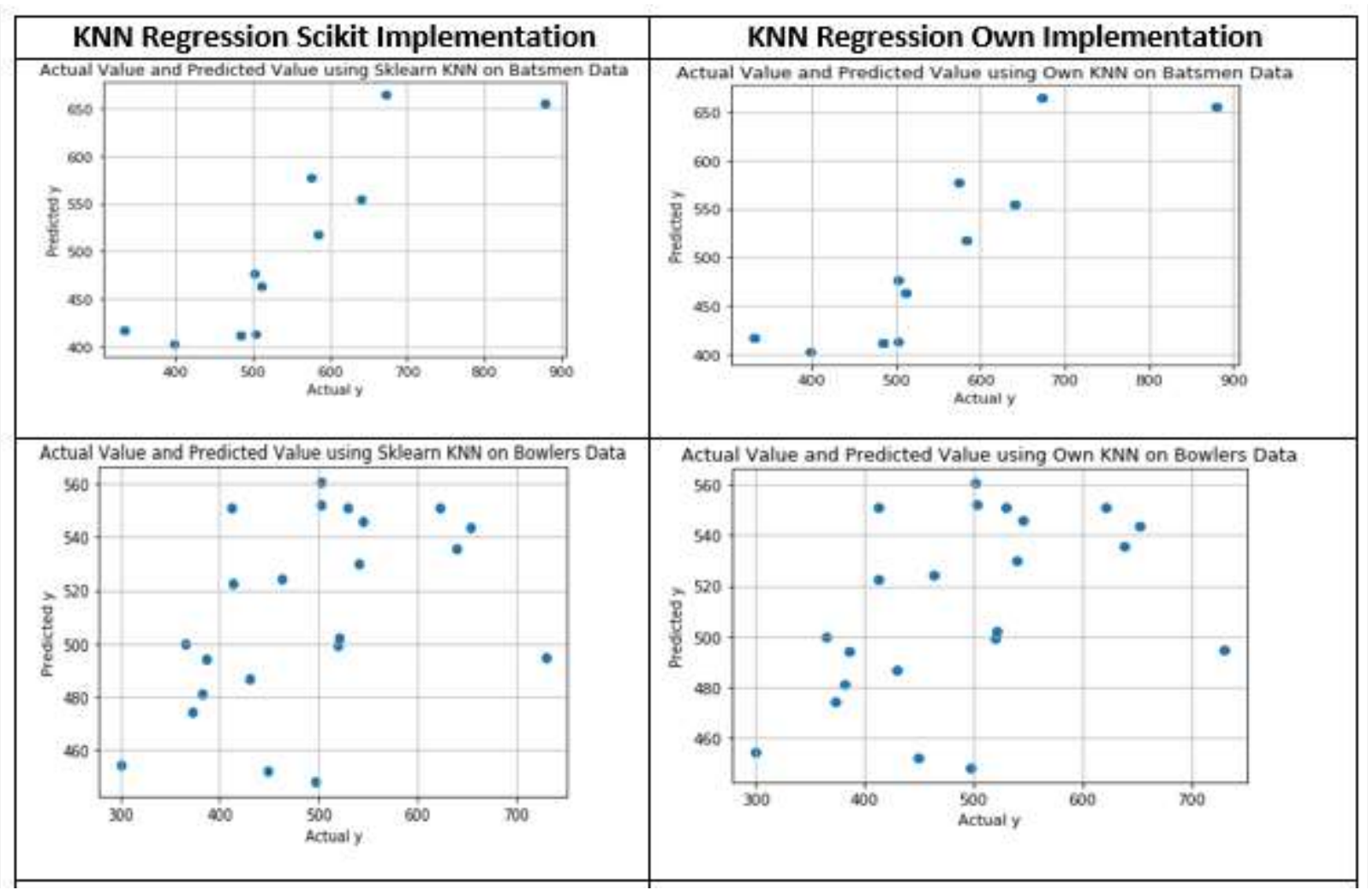

Data Visualization: The following is the data depicting the various statistics related to the project which impacts the outcome of the winning team.

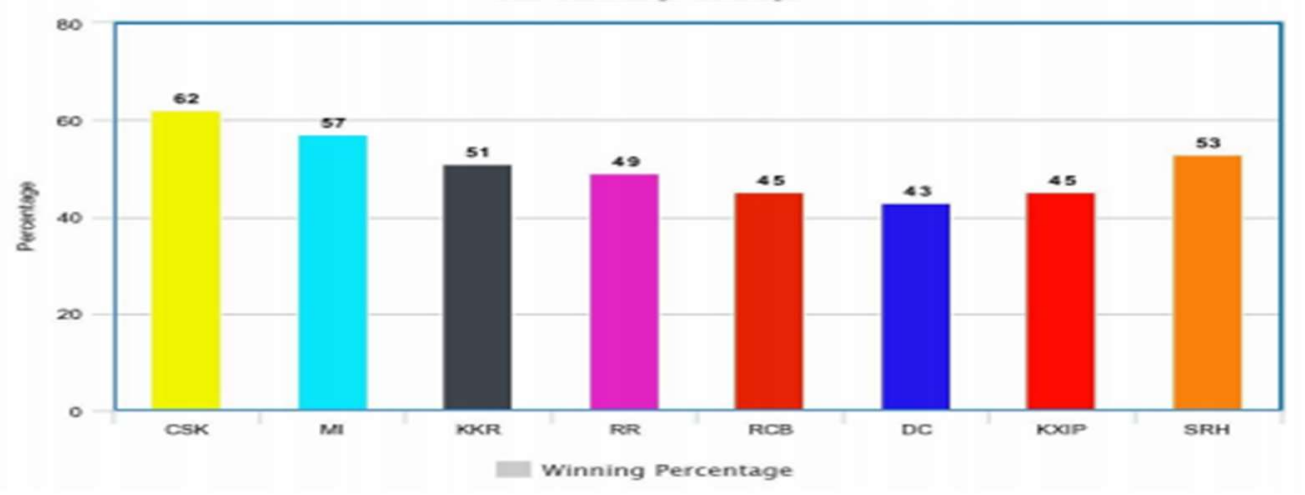

Figure 2. Team Winning Percentage 
Home Ground Advantage

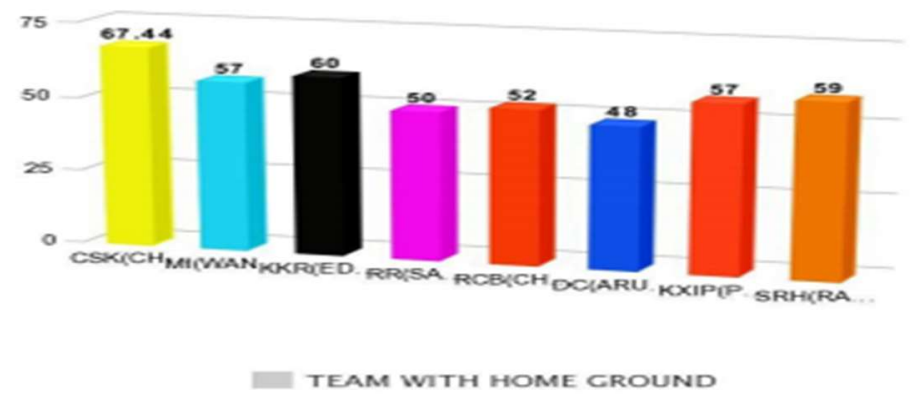

Figure 3. Home Ground Advantage

5.3.3 Teams with their IPL. Trophies

Teas wins wh troputs

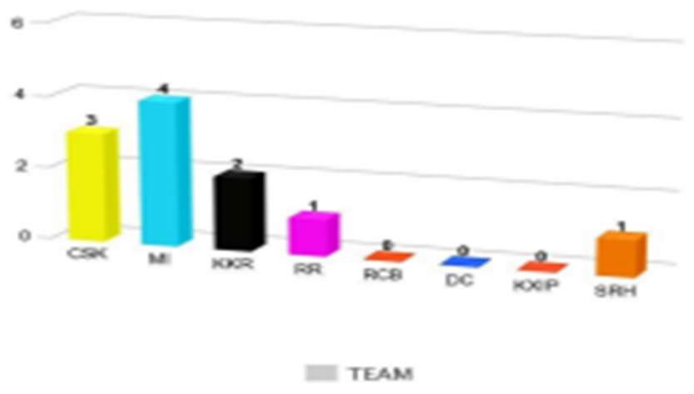

Figure 4. Teams with their IPL Trophies

5.3.4 Teams Qualifying for Playorrs

TEMUS CURUFYING FOR PLAYOFIS PEACENTNGE

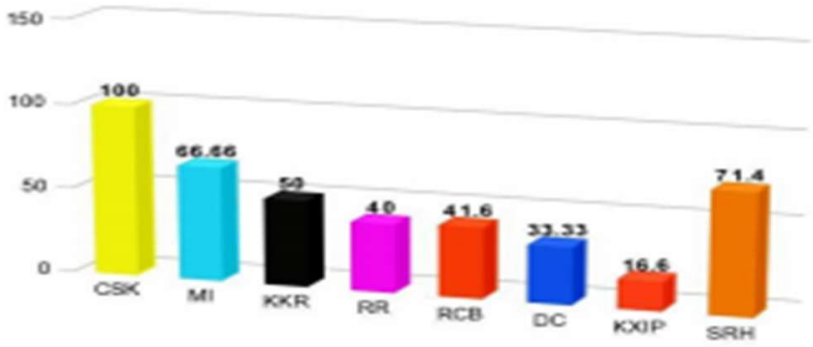


5.3.5 Individual Team Performance

SPEH WIMNINO PERCENTAOE

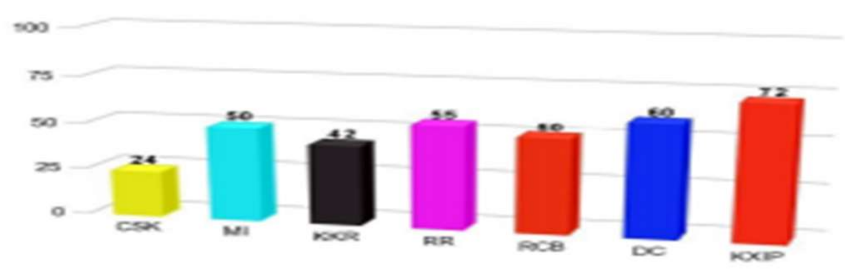

II ACAINEST TEAM

Figure 6. SRH

AR VINMINO PERCENTACE

75
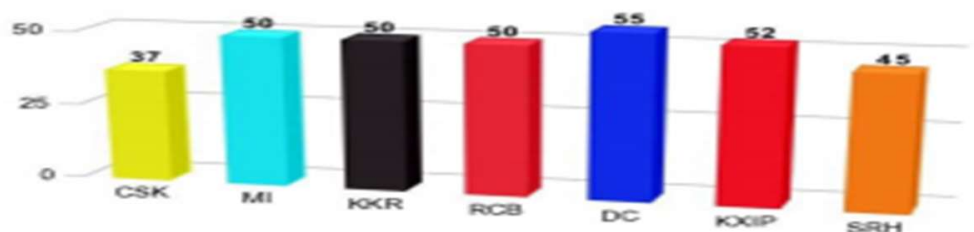

II AGAINST TEAM

Figure 7. RR

DESIGN

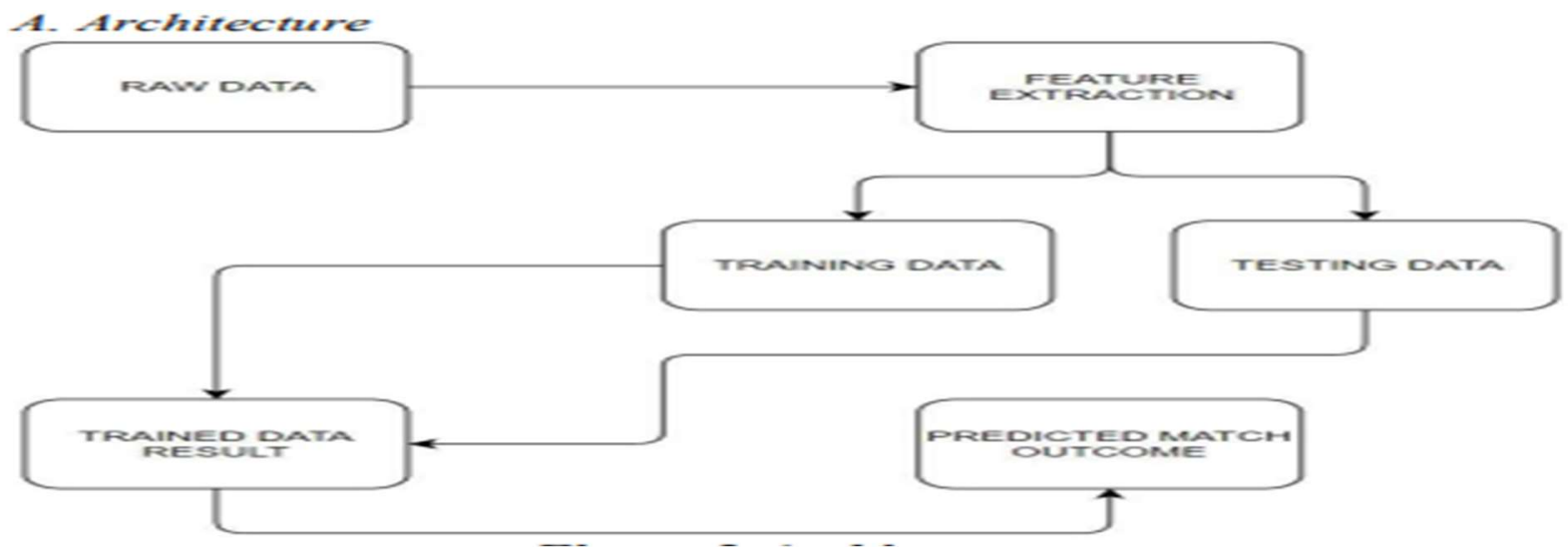


In the class diagram, there are 5 classes. The user has new data of match for prediction. The model can be trained and tested using a dataset. Match dataset contains all the features and labels. Match dataset is split into training and testing datasets.

Use Case Diagram

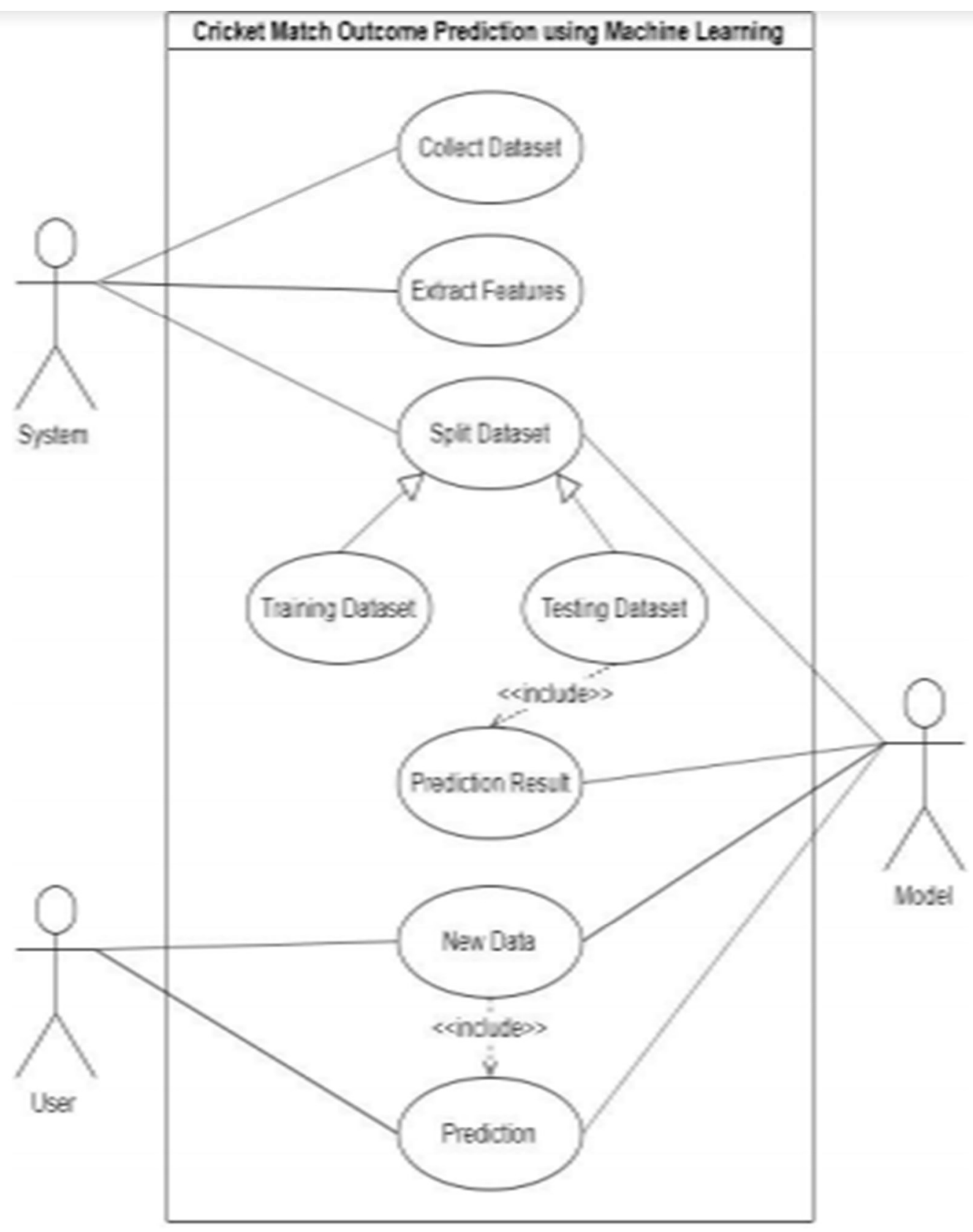


Activity Diagram

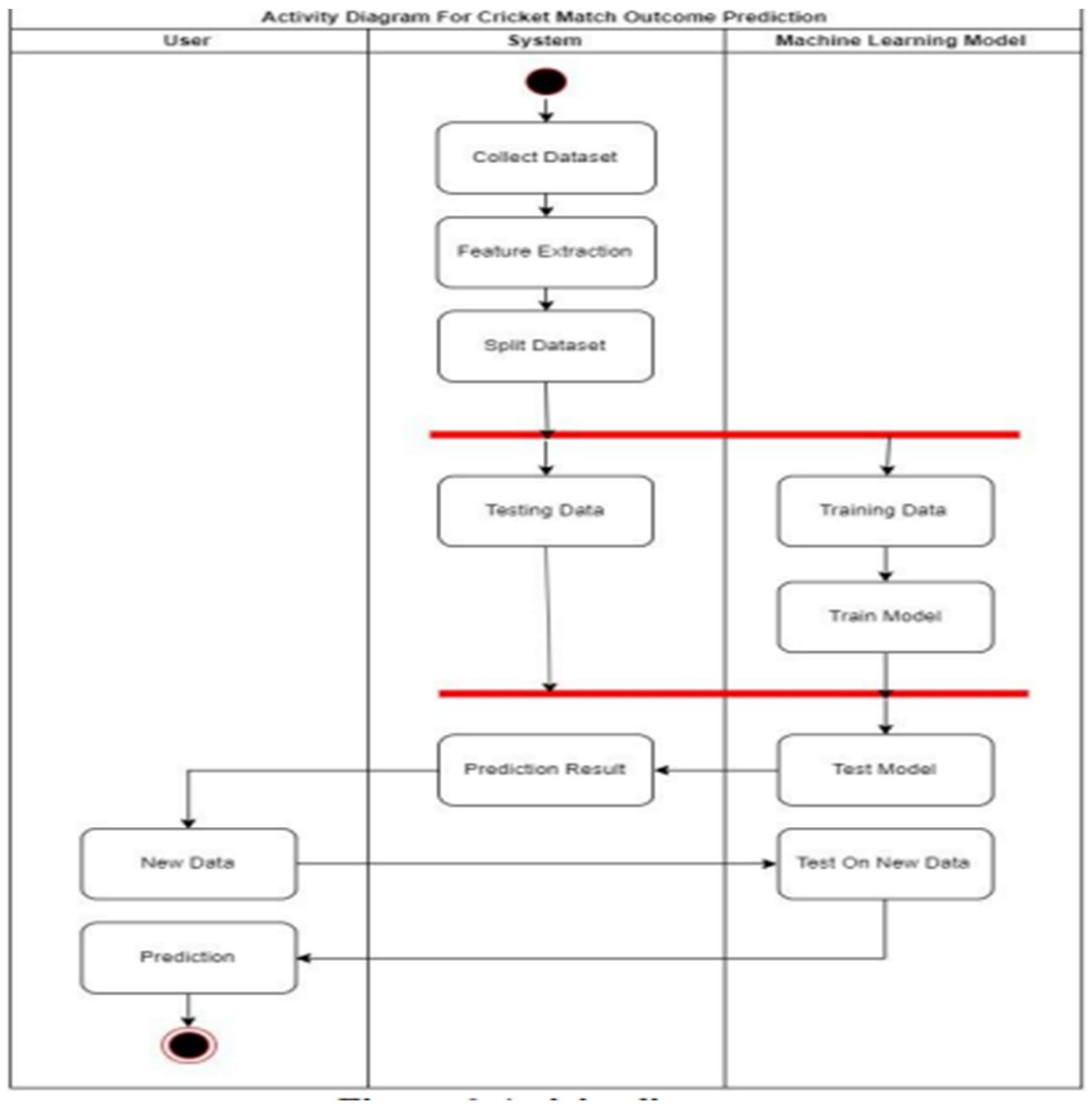

\section{RESULT}

After performing the ML algorithms, we obtained the following results based on different factors: 


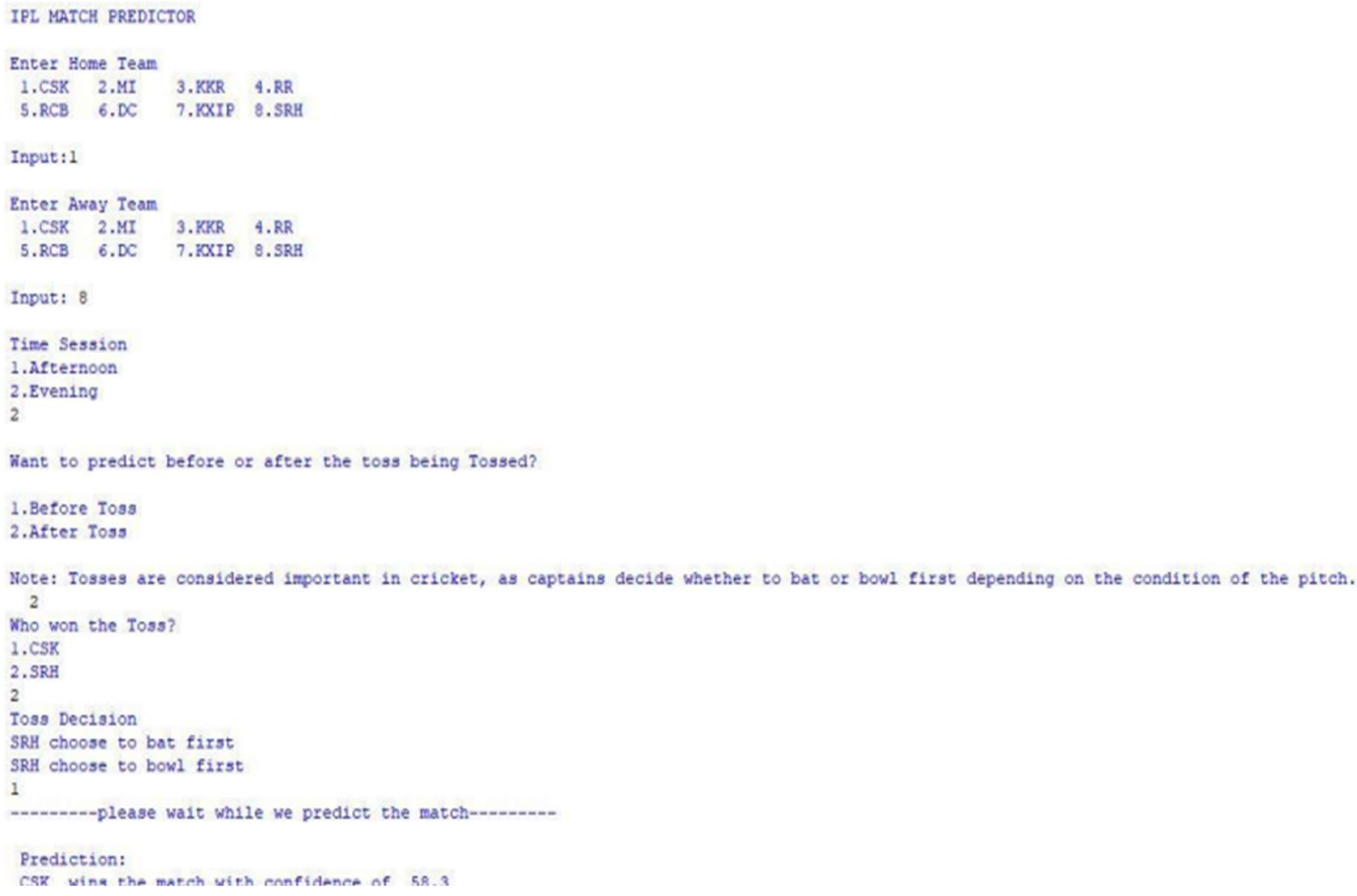

CSK vs SRH (with toss)

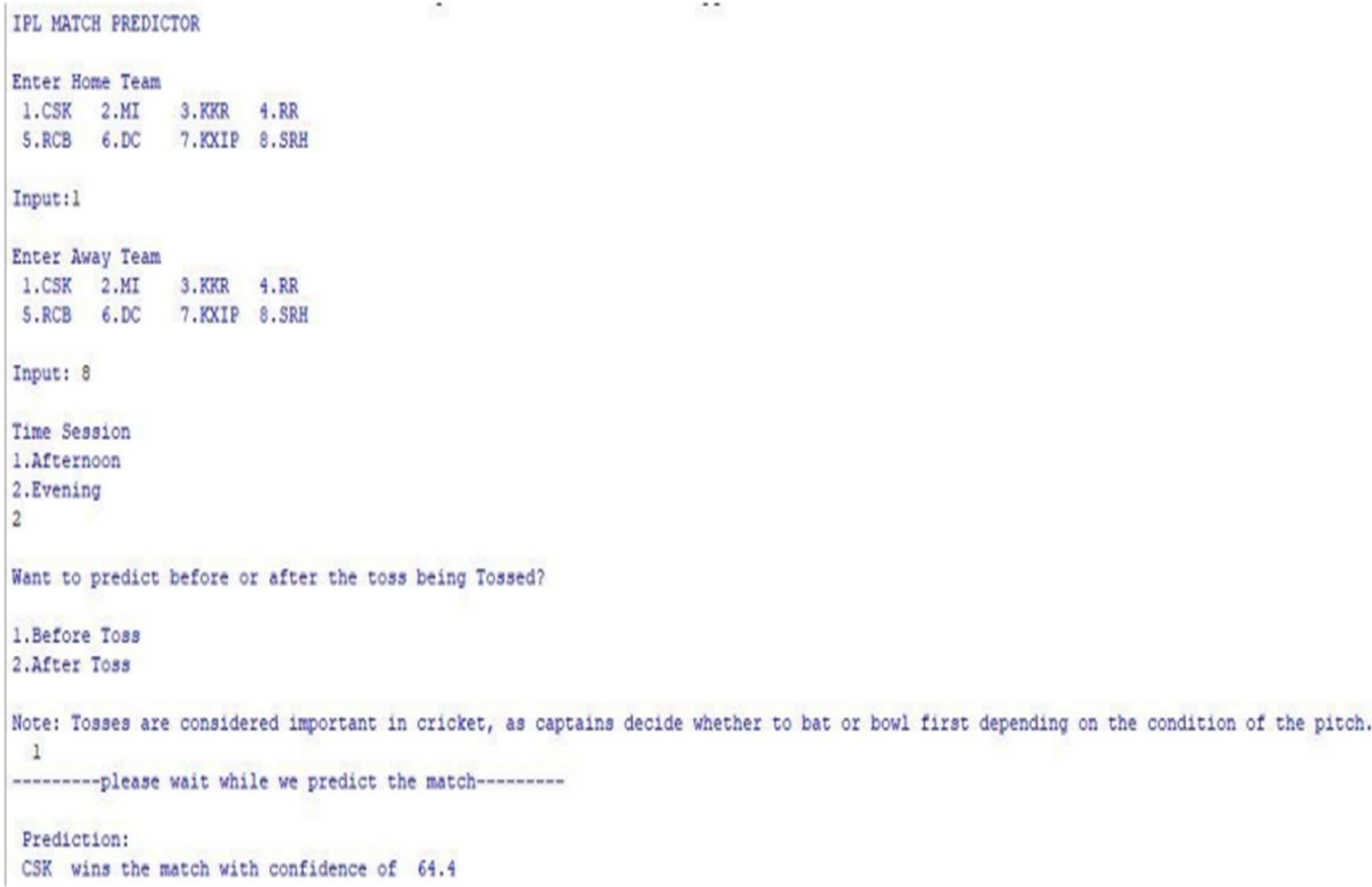


CSK vs SRH (without toss) Tosses are considered important in cricket, as captain makes the decision if the team wants to bowl or bat on the basis of pitch. from above results we can see how the toss is influencing the match outcomes.

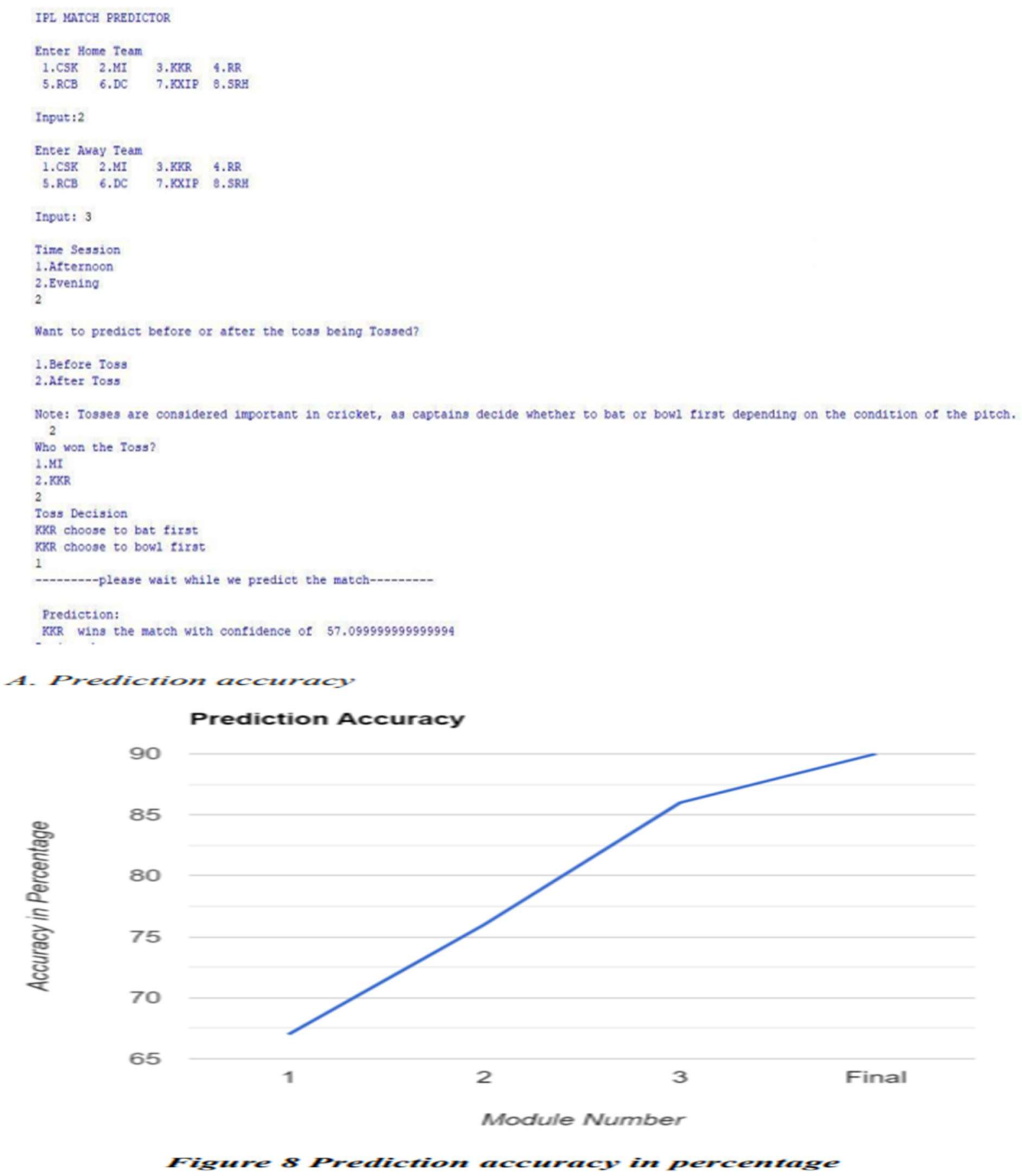

As the above graph shows the prediction accuracy of each module. We can see each module is giving more prediction accuracy $B$. Module wise prediction accuracy. 


\section{Module No of features \\ No of models \\ Prediction Accuracy}

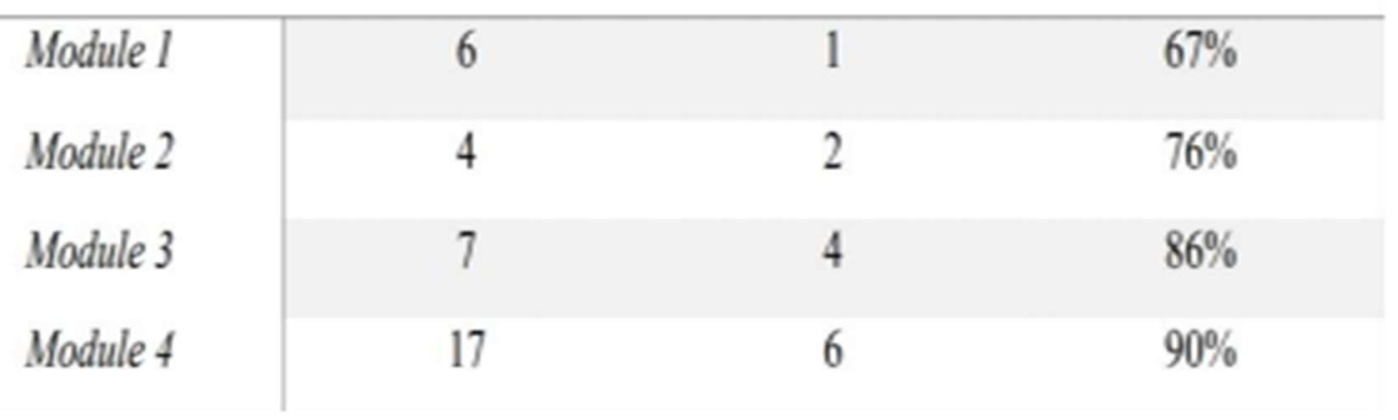

Figure 9 Module wise prediction accuracy

As the above table shows the prediction accuracy of each module and how many features are used to feed the machine learning model and how much machine learning models are used. We can see each module is giving more prediction accuracy than the previous module. We have selected 6 main key features for the first module and only one machine learning algorithm that given a prediction accuracy of $67 \%$. Then for the second module, we added one by one feature to this 6 features selected for the first module and compared the prediction accuracy with the first modules prediction accuracy, If we get the prediction accuracy greater than the first module then we keep the feature for the module 2 else we discard the feature.

\section{CONCLUSION AND FUTURE WORK}

In this paper, we selected 17 key features and 6 machine learning models that give the best possible prediction accuracy. As we can see in the below table all the papers are using a different number of features and different machine learning algorithms. Also, they are targeting different cricket formats. Some papers have only discussed features whereas some papers have discussed which machine learning algorithm will be best. The lowest accuracy is of [3] which is $63.05 \%$ and the highest accuracy is of [8] which is $85 \%$. So we analyzed every paper and found all the key factors that increased prediction accuracy and algorithms that predicted with the best accuracy. The highest prediction accuracy is $85 \%$ and in our paper, we are getting an accuracy of nearly $90 \%$. By using this model we are going to predict the outcome of twenty 20 matches, one-day international matches, and test matches also. This model can be used for predicting the outcome of other sports also like football, hockey, tennis, baseball, rugby, etc. 


\begin{tabular}{|c|c|c|c|c|}
\hline Paper No & Format & $\begin{array}{l}\text { No. or } \\
\text { Features }\end{array}$ & Algorithm & Accuracy \\
\hline [1] & T2O(IPL) & 5 & Random Forest Classifier & 68.3396 \\
\hline [2] & ODI & 10 & Logistic Regression, K-Means Clustering & - \\
\hline [3] & T20I & 16 & Non Linear Support Vector Machine & $63.05 \%$ \\
\hline [4] & ODI & 7 & - & - \\
\hline [5] & ODI & - & Bayes Classifier & $32 \%$ \\
\hline [7] & ODI & - & Support Vector Machine & $35 \%$ \\
\hline [8] & T20I & 5 & - & $85 \%$ \\
\hline [9] & T20I & - & Support Vector Machine & $83 \%$ \\
\hline This Paper & T20(CPL) & 16 & $\begin{array}{c}\text { Multiple Linear Regression, Support Vector } \\
\text { Regression, Decisice Tree Regression, } \\
\text { Random Forest Regression, Nave Bayes, } \\
\text { Logistic Regression. }\end{array}$ & $90 \% 6$ \\
\hline
\end{tabular}

From the study there are numerous elements which impact result of any IPL match is observed. Main factors that fundamentally impact any IPL match could be their host group, non-home group, arena, winner of toss and many more. This relatively helped in the calculation of strength. Different ML techniques were handed down for IPL data set which contributed to this study. The data set consists of all the IPL matches that were held from the past 6 years that is from 2014 to 2019. The prepared models were utilized to foresee the result of IPL matches. The T20 cricket has a scope for changeability, because even few balls can totally change the game. IPL was started 12 years back, there were very less number of games played compared to 50-50 and test games. Thus, structuring ML for anticipating game result with a precession of $75 \%$ is exceptionally good at this stage.

\section{References}

[1] A. L. Samuel, "Some studies in machine learning using the game of checkers. iirecent progress," in Computer Games I, pp. 366-400, Springer, 1988.

[2] A. Bandulasiri, "Predicting the winner in one day international cricket," Journal of Mathematical Sciences \& Mathematics Education, vol. 3, no. 1, pp. 6-17, 2008.

[3] Indian Premier League Official Website [4] P. Langley, W. Iba, K. Thompson, et al., "An analysis of bayesian classifiers," in Aaai, vol. 90, pp. 223-228, 1992.

[5] S. Kampakis and W. Thomas, "Using machine learning to predict the outcome of English county twenty over cricket matches," arXiv preprint arXiv:1511.05837, 2015.

[6] L. Passfield and J. G. Hopker, "A mine of information: can sports analytics provide wisdom from your data?," International journal of sports physiology and performance, vol. 12, no. 7, pp. 851-855, 2017.

[7] T. H. Davenport, "What businesses can learn from sports analytics," MIT Sloan Man-agement Review, vol. 55, no. 4, p. 10, 2014.

[8] Muhammad Yasir, LI CHEN, Sabir Ali Shah, Khalid Akbar, M.Umer Sarwar, "Ongoing Match Prediction in T20 International", International Journal of Computer Science and Network Security, Volume: 17 Number: 11 (November 2017) 
[9] A.N.Wickramasinghe, Roshan D.Yapa, "Cricket Match Outcome Prediction Using Tweets and Prediction of the Man of the Match using Social Network Analysis: Case Study Using IPL Data", International Conference on Advances in ICT for Emerging Regions, ICTer: 442 (2018)

[10] Ayush Kalla, Nihar Karle, Sushant Wagle, Sandeep Utala, “AutoPlay - Cricket Score Predictor”, International Journal of Engineering Science and Computing, Volume: 8 Issue: 4 (April 2018)

[11] Kaluarachchi, Amal, and S. Varde Aparna. "CricAI: A classification based tool to predict the outcome in ODI cricket." 2010 Fifth International Conference on Information and Automation for Sustainability. IEEE, 2010

[12] Pedregosa, Fabian, et al. "Scikit-learn: Machine learning in Python." Journal of Machine Learning Research 12.Oct (2011): 2825-2830.

[13] Sankaranarayanan, Vignesh Veppur, Junaed Sattar, and Laks VS Lakshmanan. "Auto-play: A Data Mining Approach to ODI Cricket Simulation and Prediction.” SDM. 2014

[14] "Import.io | Extract data from the web," Import.io, [Online]. Available: https://www.import.io.

[15] T. L. Saaty, "A scaling method for priorities in a hierarchichal structure," Mathematical Psychology, vol. 15, pp. 234-281, 1977.

[16] T. L. Saaty, The Analytic Hierarchy Process, New York: McGraw-Hill, 1980.

[17] N. V. Chavla, K. W. Bowyer, L. O. Hall and W. P. Kegelmeyer, "SMOTE: Synthetic Minority Oversampling Technique," Artificial Intelligence Research, vol. 16, p. 321-357, June 2002.

[18] J. Han, M. Kamber and J. Pei, Data Mining: Concepts and Techniques, 3rd Edition ed., Waltham: Elsevier, 2012.

[19] J. R. Quinlan, "Induction of Decision Trees," Machine learning, vol. 1, no. 1, pp. 81-106, 1986.

[20] J. R. Quinlan, C4.5: Programs for Machine Learning, Elsevier, 2015.

[21] L. Breiman, "Random Forests," Machine Learning, vol. 45, no. 1, pp. 5-32, 2001.

[22] T. K. Ho, "The Random Subspace Method for Constructing Decision Forests," IEEE transactions on pattern analysis and machine intelligence, vol. 20, no. 8, pp. 832-844, August 1998

[23] L. Breiman, J. Friedman, C. J. Stone and R. A. Olshen, Classification and regression trees, CRC Press, 1984.

[24] B. E. Boser, I. M. Guyon and V. N. Vapnik, "A Training Algorithm for Optimal Margin Classifiers," in Fifth Annual Workshop on Computational Learning Theory, Pittsburgh, 1992.

[25] C.-C. Chang and C.-J. Lin, "LIBSVM: A library for support vector machines," ACM Transactions on Intelligent Systems and Technology, vol. 2, no. 3, April 2011. 